\title{
The mitochondrial transporter ABC-me (ABCB10), a downstream target of GATA-1, is essential for erythropoiesis in vivo
}

\author{
BB Hyde ${ }^{1,5}$, M Liesa $^{1,5}$, AA Elorza ${ }^{1}$, W Qiu ${ }^{1}$, SE Haigh ${ }^{1}$, L Richey ${ }^{2}$, HK Mikkola ${ }^{3}$, TM Schlaeger ${ }^{4}$ and OS Shirihai ${ }^{*}, 1$
}

The mitochondrial transporter ATP binding cassette mitochondrial erythroid (ABC-me/ABCB10) is highly induced during erythroid differentiation by GATA-1 and its overexpression increases hemoglobin production rates in vitro. However, the role of $A B C$-me in erythropoiesis in vivo is unknown. Here we report for the first time that erythrocyte development in mice requires ABC-me. ABC-me- $I-$ mice die at day 12.5 of gestation, showing nearly complete eradication of primitive erythropoiesis and lack of hemoglobinized cells at day 10.5. ABC-me- $I$ - erythroid cells fail to differentiate because they exhibit a marked increase in apoptosis, both in vivo and ex vivo. Erythroid precursors are particularly sensitive to oxidative stress and ABC-me in the heart and its yeast ortholog multidrug resistance-like 1 have been shown to protect against oxidative stress. Thus, we hypothesized that increased apoptosis in ABC-me-I- erythroid precursors was caused by oxidative stress. Within this context, ABC-me deletion causes an increase in mitochondrial superoxide production and protein carbonylation in erythroid precursors. Furthermore, treatment of $A B C$-me- $I$ - erythroid progenitors with the mitochondrial antioxidant MnTBAP (superoxide dismutase 2 mimetic) supports survival, ex vivo differentiation and increased hemoglobin production. Altogether, our findings demonstrate that $\mathrm{ABC}-\mathrm{me}$ is essential for erythropoiesis in vivo.

Cell Death and Differentiation (2012) 19, 1117-1126; doi:10.1038/cdd.2011.195; published online 13 January 2012

A central event during erythroid development is the induction of the components responsible for heme biosynthesis. Although heme is also produced in non-erythroid cells, its biosynthesis is specifically regulated during erythroid differentiation. ${ }^{1-3}$ Thus, mutations in some specific genes involved in heme production have been shown to cause several human blood disorders, such as sideroblastic anemias. ${ }^{4}$ This type of anemia is characterized by abnormal erythroid differentiation with a reduction in the total number of erythrocytes. ${ }^{4}$ In addition, there are idiopathic anemias (either genetic or druginduced) in which the primary molecular defect is unknown. Recently, a novel bioinformatic approach identified new genes involved in heme biosynthesis that could be potential therapeutic targets for these disorders. ${ }^{5}$ Furthermore, this large-scale computational screen identified again the ATP Binding Cassette mitochondrial erythroid transporter (ABCme/ABCB10) as an important protein for heme biosynthesis. ${ }^{5}$

We previously identified ABC-me as a downstream target of an essential transcription factor for terminal erythroid differentiation, namely GATA- $1 .{ }^{6} \mathrm{ABC}$-me is a homodimeric transporter located in the inner mitochondrial membrane, which shows the highest expression levels in erythroid tissues. ${ }^{6,7}$ Importantly, during early stages of mouse development (day 10 post coitus, pc), ABC-me expression is detected exclusively at the primitive sites of hematopoiesis, namely the yolk sac blood islands. ${ }^{6}$ Furthermore, at day $9.5-$ $10.5 \mathrm{pc}, 95 \%$ of circulating blood cells are primitive nucleated erythroblasts (most of them basophilic and polychromatophilic) and a large number of these erythroblasts express high levels of transferrin receptor (CD71, responsible for iron uptake and required to synthesize hemoglobin) and Ter119 (membrane protein used as a marker for terminal erythroid differentiation) ${ }^{8,9}$ Therefore, maximal iron uptake and hemoglobin synthesis rates in embryonic blood cells occur at the same stage as the detection of $A B C$-me expression in the yolk sac. In addition, ABC-me (ABCB10) has been demonstrated to be required for maximal (not basal) iron import into mitochondria during in vitro erythroid differentiation, as it stabilizes the iron importer mitoferrin 1 (Mfrn1)..$^{10}$

The mitochondrial topology of $A B C$-me transporter makes it a likely candidate as a novel exporter having a role in mitochondria detoxification and/or regulating the compartmentalization of heme biosynthesis intermediates between mitochondria and cytosol. Finely tuned compartmentalization and proper mitochondrial function are not only relevant for the rates of heme production but also are essential for cell viability,

\footnotetext{
${ }^{1}$ Department of Medicine, Obesity and Nutrition section, Mitochondria ARC, Evans Biomedical Research Center, Boston University School of Medicine, Boston, MA, USA; ${ }^{2}$ Department of Laboratory Animal Medicine, Tufts University, Boston, MA, USA; ${ }^{3}$ Department of Molecular and Cellular Developmental Biology, UCLA, Los Angeles, CA, USA and ${ }^{4}$ Harvard Stem Cell Institute, Children's Hospital/Harvard Medical School, Boston, MA, USA

${ }^{*}$ Corresponding author: OS Shirihai, Department of Medicine, Obesity and Nutrition section, Mitochondria ARC, Evans Biomedical Research Center, 650 Albany Street. Room 804, Boston, MA 02118, USA. Tel: + 1617230 8570; Fax: + 1617638 7124; E-mail: shirihai@bu.edu

${ }^{5}$ These authors contributed equally to this work.

Keywords: ABCB10; mitochondria; oxidative stress; hemoglobin; erythropoiesis

Abbreviations: ABC-me, ATP Binding Cassette-mitochondrial erythroid; SOD, superoxide dismutase; ES, embryonic stem cells; pc, post coitus; ROS, reactive oxygen species; MnTBAP, Mn(III)tetrakis(4-benzoic acid)porphyrin; NAC, N-acetyl-cysteine; FITC, fluorescein isothiocyanate; PE, phycoerythrin; TUNEL, terminal deoxynucleotidyl transferase dUTP nick end labeling; BrdU, bromodeoxiuridine; BCL-xL, B-cell lymphoma extra large; Mfrn1, mitoferrin 1

Received 31.3.11; revised 22.11.11; accepted 29.11.11; Edited by R De Maria; published online 13.1.12
} 
as accumulation of heme intermediates or regulators of their production in cytosol and/or mitochondria can lead to oxidative stress and toxicity. ${ }^{1,2,4,11}$ In this regard, ABC-me overexpression in erythroid derived cells (murine erythroleukemia cells) increases both the total levels and production rates of hemoglobin, without affecting the shape or the number of mitochondria. ${ }^{6}$ Furthermore, ABC-me and its yeast ortholog multidrug resistance-like 1 were shown to protect from increased mitochondrial oxidative stress caused by ischemiareperfusion in the heart or by atm deletion in yeast, respectively. ${ }^{12-14}$ Altogether, these data highlight an important role of ABC-me transporter in heme and hemoglobin synthesis and in protection from oxidative stress. However, the role of $A B C$-me in erythroid differentiation in vivo, the effects of $A B C$-me loss-offunction on hemoglobin synthesis and oxidative stress in erythroid cells and whether ABC-me is necessary for erythroid development still remained unknown until now.

Here we show that mice lacking $A B C-m e(A B C-m e-/-)$ are embryonic lethal at day $12.5 \mathrm{pc}$. ABC-me-/-embryos show a significant defect in primitive erythroid development presenting a cardiovascular system empty of red blood cells at day $10.5 \mathrm{pc}$, before definitive erythropoiesis starts in the liver. We show that $A B C-m e-/-$ erythroid precursors $\left(C D 71^{+}\right)$undergo apoptosis at day 10.5 pc or during ex-vivo differentiation of embryonic blood progenitors or embryonic stem cells (ES cells). Mitochondrial oxidative stress contributes to ABC-me-/- erythroid precursor apoptosis, as both their survival and hemoglobin levels are increased by the mitochondrial antioxidant MnTBAP (superoxide dismutase 2 mimetic). In all, we demonstrate that ABC-me is essential for erythroid development and that its loss-of-function reduces hemoglobin production, increases oxidative stress and apoptosis in erythroid cells.

\section{Results}

Generation and characterization of ABC-me-Imouse. During embryogenesis, ABC-me expression is found exclusively in the erythroid precursors found in the yolk sac blood islands on embryonic day 10 pc, which are the primitive sites of hematopoiesis. ${ }^{6}$ Given this embryonic pattern of $\mathrm{ABC}$-me expression, a global knockout mouse model was used to study the role of $A B C$-me in hematopoiesis. $A B C$ me $+/$ - mice were generated in a C57BI6/129SvEvBrd mixed background by replacing $A B C-$ me exons 2 and 3 with an insert containing a neomycin-resistance cassette (a strategy designed and executed by Lexicon Genetics, The Woodlands, TX, USA; today Taconic). ${ }^{13}$ ABC-me $+/-$ mice were backcrossed onto C57BI6 background and bred to generate ABC-me-/- mice. Western blot analyses show no $A B C-m e$ expression in day 10.5 embryonic ABC-me-/- blood lysates (see Supplementary Figure S1 and Liesa et al. ${ }^{13}$ ).

ABC-me-I- mice were embryonic lethal and ABC-me + Imice were viable, with no obvious phenotype in the post-natal life under basal conditions. ${ }^{13}$ Given that embryonic lethality caused by severe anemia was previously detected in day 10.5-11.5 pc GATA-1-/- embryos (ABC-me is a downstream target of GATA-1), we decided to investigate $A B C$ me-/- embryo development between days 10.5 and $13.5 \mathrm{pc}$. ABC-me-/- embryos were found to be dead and smaller at day $12.5 \mathrm{pc}$, as determined by the absence of a visually detectable heartbeat (Table 1). ABC-me-/- embryos were

Table 1 Numbers of ABC-me-/- embryos and their phenotype

Day 10.5

\begin{tabular}{|c|c|c|c|c|c|c|}
\hline \multirow{2}{*}{$\begin{array}{l}\text { Phenotype } \\
\text { Genotype }\end{array}$} & \multicolumn{3}{|c|}{ Hemoglobinized (alive) } & \multicolumn{3}{|c|}{ Non-hemoglobinized (alive) } \\
\hline & WT & ABC-me+l- & ABC-me-I- & WT & ABC-me+l- & ABC-me-I- \\
\hline \multicolumn{7}{|l|}{ Embryos } \\
\hline $\begin{array}{l}107 \text { (11 Litters) } \\
\% \text { Detected } \\
\% \text { Expected }\end{array}$ & $\begin{array}{l}22 \\
20.5 \\
25\end{array}$ & $\begin{array}{l}55 \\
51.4 \\
50\end{array}$ & $\begin{array}{c}3^{a} \\
2.8^{a} \\
25\end{array}$ & $\begin{array}{l}0 \\
0 \\
0\end{array}$ & $\begin{array}{c}4 \\
3.7 \\
0\end{array}$ & $\begin{array}{l}23 \\
21.5 \\
0\end{array}$ \\
\hline
\end{tabular}

Day 12.5

\begin{tabular}{|c|c|c|c|c|c|c|}
\hline \multirow{2}{*}{$\begin{array}{l}\text { Phenotype } \\
\text { Genotype }\end{array}$} & \multicolumn{3}{|c|}{ Alive (heart beat) } & \multicolumn{3}{|c|}{ Dead (no heart beat) } \\
\hline & WT & ABC-me+l- & ABC-me-I- & WT & ABC-me+l- & ABC-me-I- \\
\hline \multicolumn{7}{|l|}{ Embryos } \\
\hline $\begin{array}{l}40 \text { (5 Litters) } \\
\% \text { Detected } \\
\% \text { Expected }\end{array}$ & $\begin{array}{l}10 \\
25 \\
25\end{array}$ & $\begin{array}{l}13 \\
32.5 \\
50\end{array}$ & $\begin{array}{r}0 \\
0 \\
25\end{array}$ & $\begin{array}{c}1^{b} \\
2.5^{b} \\
0\end{array}$ & $\begin{array}{c}6^{\mathrm{b}} \\
15^{\mathrm{b}} \\
0\end{array}$ & $\begin{array}{c}10^{c} \\
25^{c} \\
0\end{array}$ \\
\hline
\end{tabular}

Day 13.5

\begin{tabular}{lccc}
\hline Phenotype & \multicolumn{3}{c}{ Alive (heart beat, non-resorbed) } \\
\cline { 2 - 4 } Genotype & WT & ABC-me+l- & ABC-me-I- \\
\hline Embryos & 11 & 16 & 0 \\
27 (6 Litters) & 7 & 59.3 & 0 \\
$\%$ Detected & 25 & 50 & 25 \\
$\%$ Expected & & &
\end{tabular}

${ }^{a}$ Much paler than wild type, but some hemoglobin could be seen by naked eye. ${ }^{\mathrm{b}}$ These seven dead embryos (WT and ABC-me+/-) were detected in the same female (one out of the five litters). ' Smaller, partially resorbed 
completely resorbed by the uterus by day $13.5 \mathrm{pc}$ (Table 1). Upon pathological examination, ABC-me-/- embryos presented severe anemia at day $10.5 \mathrm{pc}$, as shown by the lack of red coloring of the yolk sac vasculature (Table 1, Figures 1a and b). Histological examination and hematoxylin and eosin (H\&E) staining revealed that ABC-me-/- day 10.5 pc yolk sac blood islands show a significant reduction in the total number of cells, as well as few cells with pyknotic nuclei (Figures $1 \mathrm{c}$ and $\mathrm{d}$ ).

Effects of ABC-me loss-of-function on erythroid precursors cell death. The histological findings in $A B C$ me-l- yolk sac blood islands suggested an increase of cell death in erythroid precursors. To test this hypothesis, frozen sections of day $10.5 \mathrm{pc}$ embryos with intact yolk sac were stained with DAPI to detect nucleated cells (Figures 2a and b) and terminal deoxynucleotidyl transferase dUTP nick end labeling (TUNEL)-bromodeoxiuridine (BrdU) to detect apoptotic cells (Figures $2 \mathrm{c}$ and $\mathrm{d}$ ). An abnormal low number of nucleated cells were detected in ABC-me-/yolk sac blood islands, which is in marked contrast with wildtype (WT) embryos (Figures $2 a$ and b). There was little or no TUNEL-BrdU detection in WT blood islands (Figure 2c), whereas most ABC-me-/- cells show positive TUNEL-BrdU staining indicative of activation of apoptosis (Figure $2 \mathrm{~d}$ ). These results were also confirmed by analysis of activated caspase 3 and an earlier marker of apoptosis, Annexin V, in WT and ABC-me-l- yolk sacs (Supplementary Figure S2).

To corroborate increased apoptosis in erythroid precursors, isolation of circulating blood from WT, ABC-me $+/-$ and
ABC-me-/- yolk sacs and embryos (day $10.5 \mathrm{pc}$ ) was performed. After isolation, blood cells were co-labeled with a phycoerythrin (PE)-conjugated anti-CD71 antibody (transferrin receptor, a surface marker of erythroid precursors) and TUNEL-BrdU, which was detected with an anti-BrdU antibody conjugated to fluorescein isothiocyanate (FITC). Doublelabeled blood cells were analyzed by flow cytometry, and TUNEL-BrdU (FITC) signal was only quantified in CD71positive cells. Most erythroid precursors from ABC-me-/embryos were apoptotic (72\%), whereas only 10 and $20 \%$ of WT and ABC-me+/- CD71-positive cells, respectively, showed detectable TUNEL-BrdU signal (Figure 2e). These findings were also confirmed by propidium iodide (PI) staining (75\% of cell death in ABC-me-/- versus an approximate $40 \%$ in WT and ABC-me $+/-$ ) and caspase 3 activation (30\% in ABC-me-/- and $15 \%$ in WT and ABC-me $+/-$ ) in erythroid precursors (Figures $2 f$ and $g$ ).

Effects of $A B C$-me loss-of-function in primitive erythroid differentiation. Primitive and definitive erythropoiesis can be tracked by measuring CD71 together with Ter119 (Figure 3a). ${ }^{8,15}$ ABC-me-/- embryos are severely anemic at day 10.5 pc (Figure 1), when $95 \%$ of circulating blood cells are primitive nucleated erythroblasts in WT embryos. ${ }^{8}$ Furthermore, a large percentage of these circulating blood cells $\left(70-80 \%\right.$ at day $9.5 \mathrm{pc}^{8}$; around $30-40 \%$ at day 10.5 ; (Figures $3 a$ and b)) harbor high levels of expression and are positive for both CD71 $\left(\mathrm{CD} 71^{+}\right.$high $)$and Ter119 (Ter119+ high). Interestingly, in adult definitive erythropoiesis models (i.e., bone marrow), CD71 ${ }^{+}$high cells
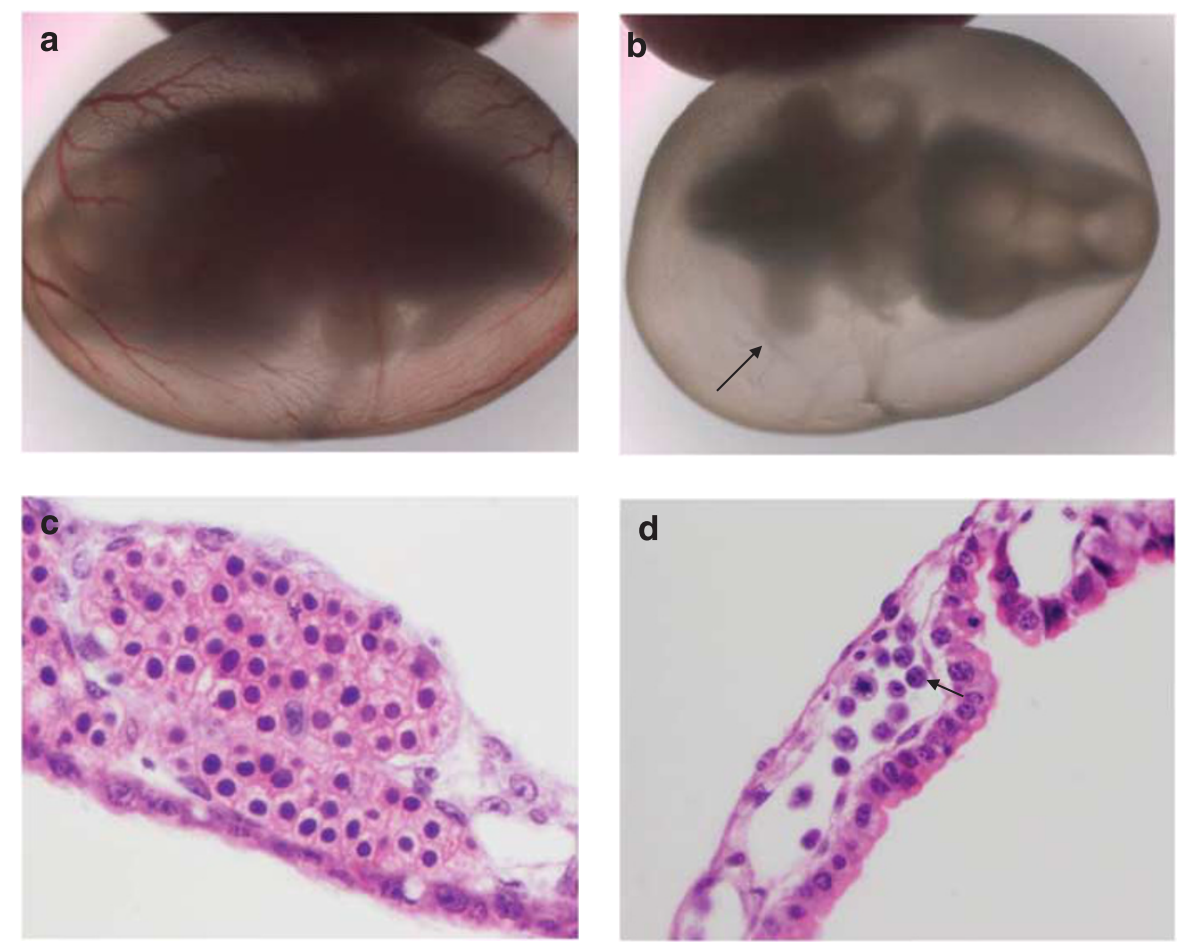

Figure $1 \mathrm{ABC}$-me deletion results in severely anemic embryos at day $10.5 \mathrm{pc}$. (a) Wild-type and (b) ABC-me-/- intact embryos (day $10.5 \mathrm{pc}$ ) light microscopy images. Note that in (b) ABC-me-/- embryo is not hemoglobinized, whereas the vasculature of the yolk sac is present (black arrow). HE-stained sections of yolk sac blood islands from (c) WT and from (d) ABC-me-l- day $10.5 \mathrm{pc}$ embryos. Note a significant reduction in erythroid cells and the remaining cells showing pyknotic nuclei only in (d) $\mathrm{ABC}-\mathrm{me}-1-$, as pointed to by the arrow ( $n=5$ embryos) 

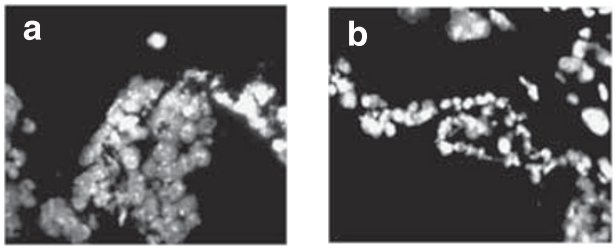

e
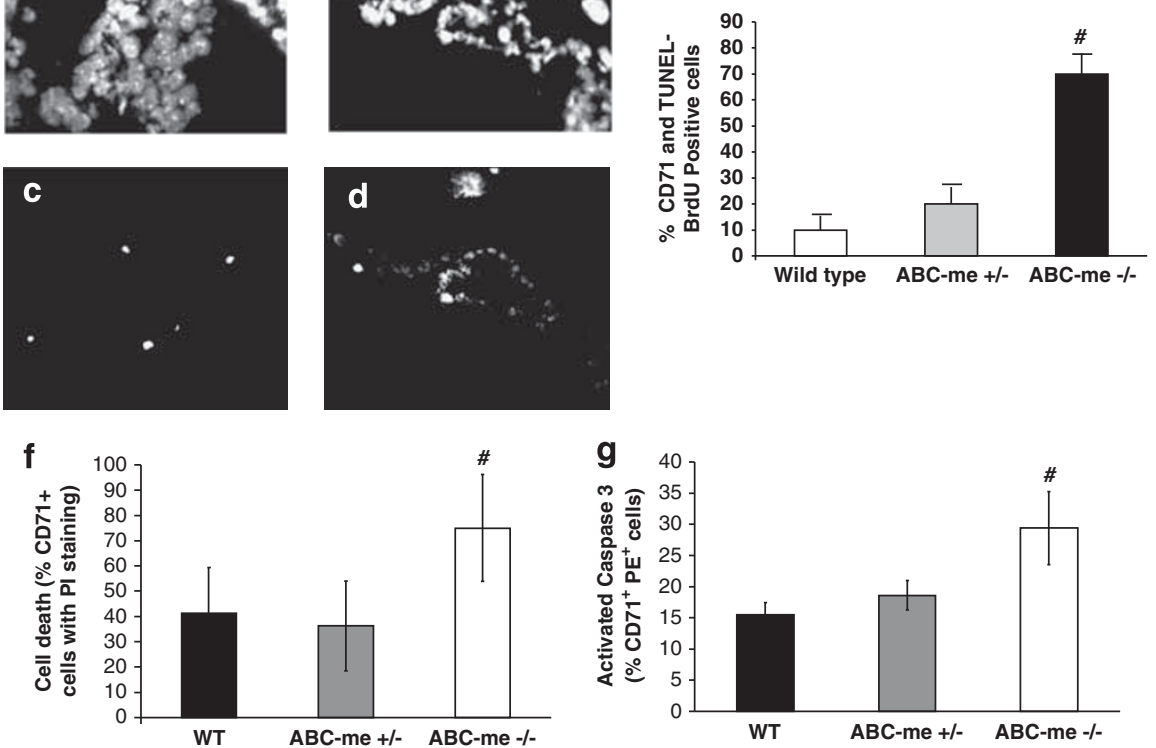

Figure 2 ABC-me-1- embryos display higher level of apoptosis in erythroid precursors. (a-d) Representative images of the yolk-sac blood islands obtained from ( $\times 40$, epi-fluorescence microscope) frozen sections from day $10.5 \mathrm{pc}$ embryos co-stained with DAPI and TUNEL-BrdU. (a) Wild-type sections show high number of cells (DAPI nuclear staining), whereas (b) ABC-me-/- sections show a reduction in cell number within the yolk-sac blood island. (c) Wild-type blood islands displayed minimal TUNELBrdU signal, whereas (d) ABC-me-/- yolk sac blood islands displayed higher levels of TUNEL-BrdU signal. (e-g) Quantification of flow cytometry analysis of isolated embryonic blood from WT and ABC-me-/- day 10.5 pc embryos. (e) Cells were co-stained with CD71 (conjugated to PE) and with BrdU (conjugated to FITC) antibodies. Percentage of TUNEL-BrdU-positive cells was quantified in cells positively gated for CD71 (transferrin receptor), as a marker for the erythroid lineage. (f) Cells co-stained with CD71 antibody conjugated to FITC and PI, representative of dead cells. Percentage of PI-positive cells was quantified in cells positively gated for CD71. (g) Cells were co-stained with CD71 antibody conjugated to FITC and FLICA-PE fluorescent substrate activated by caspase 3-mediated cleavage. Percentage of FLICA-positive cells was quantified in cells positively gated for CD71. Statistically significant differences are shown: ABC-me-l-CD71 ${ }^{+}$cells versus WT (\#); $n=3$ independent experiments; $1 \times 10^{5}$ cells per sample; $P \leqslant 0.05$ ANOVA. Error bars represent standard deviation

mostly correspond to basophilic and polychromatophilic erythroblasts, in which hemoglobin synthesis rates are maximal $^{15}$ (Figure $3 \mathrm{a}$ ). The same holds true for primitive embryonic erythropoiesis. ${ }^{8}$

In Figures $3 a$ and $b$, we have defined five regions (R1-R5) of a flow cytometry scatter plot according to the expression levels of CD71 and Ter119 in circulating blood cells from day 10.5 embryos. The majority of these cells in day $10.5 \mathrm{WT}$ embryos are CD71 ${ }^{\text {thigh }}$ (mostly located in R3, few in R2), consistent with a majority of polychromatophilic and basophilic erythroblasts at this stage in embryonic blood (Figures $3 a, b$ and $c$ and Fraser et $a l^{8}$ ) Interestingly, the R3 population (the one harboring the highest levels of CD71 expression and therefore high hemoglobin synthesis rates) is almost absent in ABC-me-1- embryos (Figures $3 b$ and $c$ ). In addition, the R3 population is also reduced (but to a lesser extent) in ABCme $+/-$ embryos (Figures $3 b$ and $c$ ). However, this reduction does not cause any lethality ${ }^{13}$ or pathologic anemia in ABCme $+/$ - embryos or during their post-natal life under basal conditions (data not shown). Thus, these data together with the apoptosis measurements (Figure 2) suggest that R3 population (mostly polychromatophilic erythroblasts) is reduced due to increased apoptosis in $\mathrm{R} 2$ and $\mathrm{R} 3$ populations and/or during transition from R2 to R3 (CD71 + high cells).

To further confirm that differentiation is stalled during the peak of hemoglobin synthesis (R2 and R3 populations), benzidine and Okajima stainings were performed in fixed sections of the yolk sac and in isolated blood cells from WT and ABC-me-/- day 10.5 pc embryos (Figure 3d). Both staining methods revealed, in immunohistological sections, a significant reduction in hemoglobinized cells in ABC-me-/yolk sac blood islands, when compared with the WT (Figure 3d). Quantification of cytospine- and benzidinestained embryonic blood cells revealed that only $8 \%$ of ABC-me-I- isolated blood cells were hemoglobinized, in marked contrast with the $35-40 \%$ detected in WT and ABC-me $+1-$ blood cells (Figure 3e). Furthermore, May-Grumwald-Giemsa staining revealed less hemoglobinized erythroid precursors in ABC-me-/- cells (most of them showing the size and morphology of basophilic erythroblasts, R2, nucleated but with a bigger cytoplasm; Figure 3d). This phenotype was again in marked contrast with WT blood cells, where a higher number of circulating blood cells displayed the size and morphology characteristic of polychromatophilic erythroblasts (nucleated, with a darker color and a smaller cytoplasm, R3; Figure 3d).

Increased ROS and oxidative stress caused by the lack of ABC-me-I- triggers apoptosis and the concomitant deficiency in erythrocyte development. Defects in mitochondrial detoxification, hemoglobin synthesis and/or in the compartmentalization of its intermediates are known to 
a

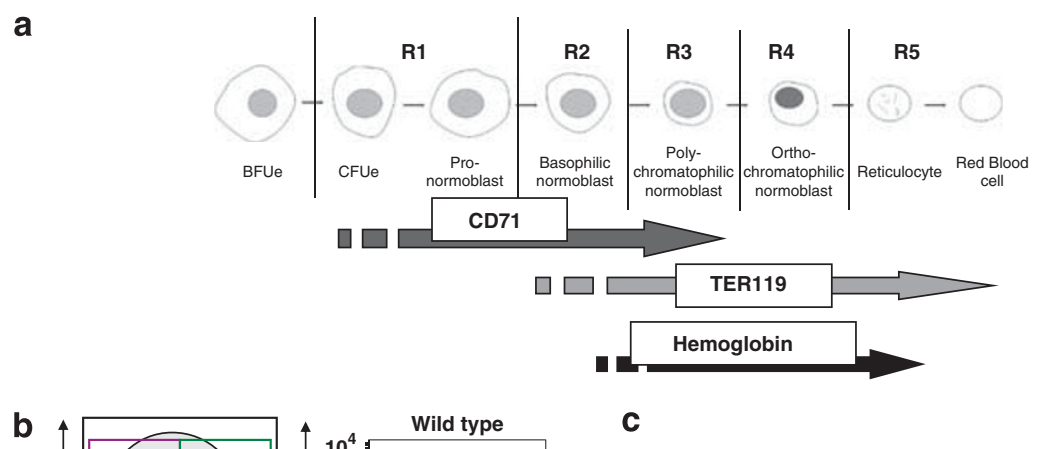

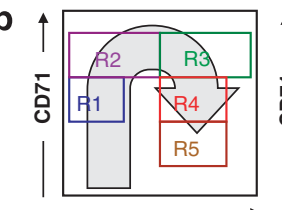
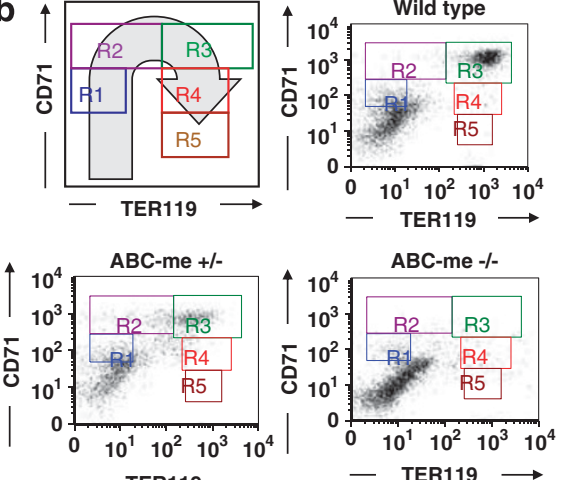

- TER119

- TER119 $\longrightarrow$

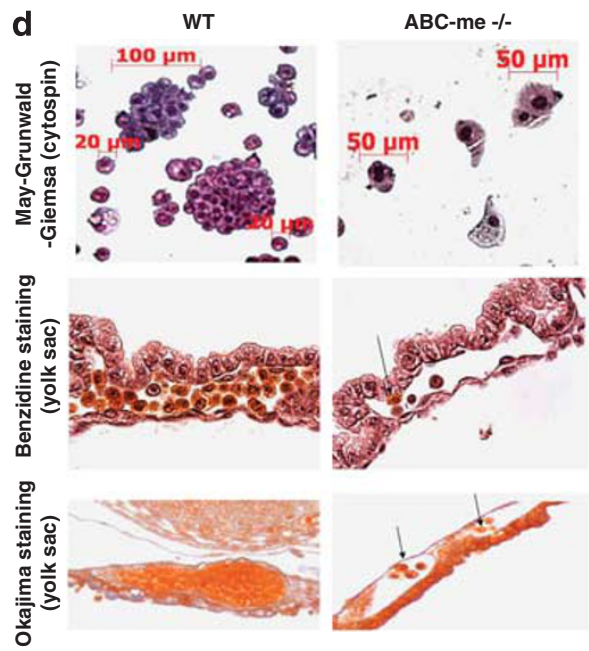

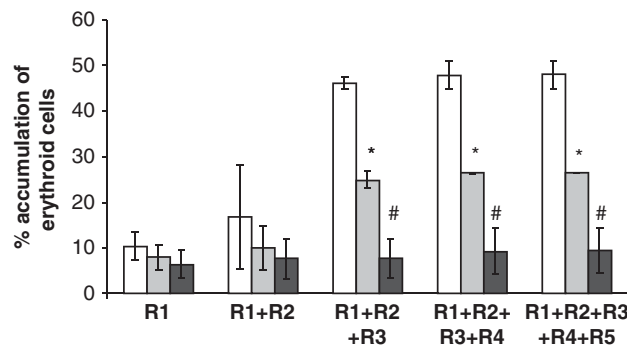

e

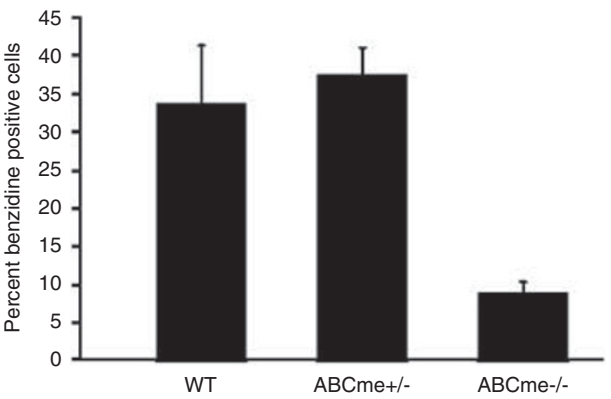

Figure 3 Effects of ABC-me loss-of-function in primitive erythroid differentiation. The expression levels (as determined by flow cytometry) of CD71 and TER119, allows for the identification and gating of five regions (from R1 to R5) within a flow cytometry scatter plot. These five regions contain distinct cell types found in the different stages of erythrocyte differentiation (as described in Kina et al..$^{9}$ and Zhang et al. ${ }^{15}$ ). (a) CFU-E and proerythroblasts are mostly located in R1, basophilic erythroblasts mostly in R2, polychromatophilic erythroblasts mostly in R3, orthochromatophilic erythroblasts mostly in R4, reticulocytes and erythrocytes in R5. (b) Representative flow cytometry scatter plot of isolated embryonic blood from day $10.5 \mathrm{pc}$ embryos analyzed for CD71 (antibody conjugated to FITC) and Ter119 (antibody conjugated to PE). ABC-me-I- blood is deficient in R3 (hemoglobinized erythroblasts) as compared with WT. (c) Quantification of erythroid progenitor and precursor populations in isolated embryonic blood (day 10.5 pc). ABC$\mathrm{me}+I_{-}\left(^{*}\right)$ and ABC-me-I- (\#) embryonic blood show a reduction in R3 hemoglobinized erythroblasts in comparison with WT R3 population $\left(n=3\right.$ experiments; $1 \times 10^{5}$ cells per sample; $P<0.05$ ANOVA). Error bars are representative of standard deviation. (d) Isolated blood from day $10.5 \mathrm{pc}$ embryos was cytospun and stained with May-GrunwaldGiemsa. Wild type blood cells demonstrated size and morphology consistent with normal erythroid precursors, mostly nucleated polychromatophilic normoblasts. Isolated ABC-me-I- cells contained a low number of erythroid precursors, with a size and morphology indicative of a less differentiated population of primarily basophilic normoblasts (larger nuclei and cytoplasm, see red scale bars representing the diameter in $\mu \mathrm{m}$ of the cells and the colonies, respectively). Sections of day $10.5 \mathrm{pc}$ embryonic yolk sac were stained both with $\mathrm{H \& E} /$ benzidine and with Okajima hemoglobin stain to detect the presence of hemoglobin $(n=5$ mice for all groups and slides were examined by multiple investigators at $\times 40$ magnification). (e) Percent benzidine-positive cells in blood isolated from $10.5 \mathrm{pc}$ embryos $(n=5$ mice per group). Error bars represent standard deviation

cause and exacerbate oxidative stress. ${ }^{1,3,4,16}$ Given the role of $\mathrm{ABC}-\mathrm{me}$ in hemoglobin synthesis and the protection from increased mitochondrial oxidative stress in cardiac cells and yeast, ${ }^{12,13}$ we hypothesized that lack of ABC-me could be triggering oxidative stress in erythroid cells. A reliable marker of oxidative stress is protein carbonylation, which results from protein oxidation by reactive oxygen species (ROS). Mitochondrial protein fractions were obtained from day 10.5 pc embryonic blood and derivatized in order to measure protein carbonylation by western blot (see Materials and Methods). ABC-me-l- mitochondria showed a fourfold increase in carbonylated proteins compared with WT 
mitochondria (Figures $4 a$ and $b$ ). Furthermore, the band pattern of carbonylated proteins was different in ABC-me-/mitochondria when compared with WT or ABC-me+/mitochondria, suggesting increased oxidative damage of some specific mitochondrial proteins in ABC-me-/precursors (Figure 4a). Interestingly, protein carbonylation was not increased in total lysates from ABC-me-/embryos, suggesting that increased oxidative damage is specific and only detectable in the mitochondrial compartment of circulating blood cells (data not shown).

Increased levels of oxidized proteins caused by ABC-me deficiency may be the result of increased ROS production. To test this possibility, day 10.5 circulating blood cells were co-labeled with the mitochondrial matrix ROS probe Mitosox (which detects superoxide) and FITC-conjugated anti-CD71 antibody. ABC-me-/- CD71 ${ }^{+}$blood cells showed an increased percentage of cells with detectable levels of superoxide (72\% ABC-me-/- versus $32 \%$ WT CD71 ${ }^{+}$cells positive for Mitosox staining; Figures $4 \mathrm{c}$ and d). In addition, the majority of ABC-me-/- CD71 ${ }^{+}$erythroid cells had higher levels of mitochondrial ROS, as measured by Mitosox fluorescence intensity (68\% ABC-me-l- versus 18\% WT and $34 \% \mathrm{ABC}-\mathrm{me}+\mathrm{I}-\mathrm{CD} 71^{+}$cells; Figures $4 \mathrm{c}$ and $\left.\mathrm{e}\right)$.

To determine the contribution of increased ROS levels to ABC-me-/- erythroid development phenotype, we tested the effect of treatment with antioxidants on erythropoiesis. As ABC-me-/- erythroid cells show an increase in mitochondrial superoxide, a superoxide dismutase 2 (SOD2) mimetic compound (MnTBAP) was used to reduce mitochondrial superoxide levels. ${ }^{17}$ Isolated blood cells from day 10.5 pc embryos were differentiated in a hematopoietic semi-solid media (see Materials and Methods). The number of the

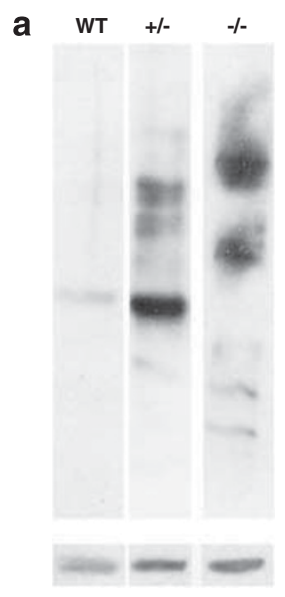

b
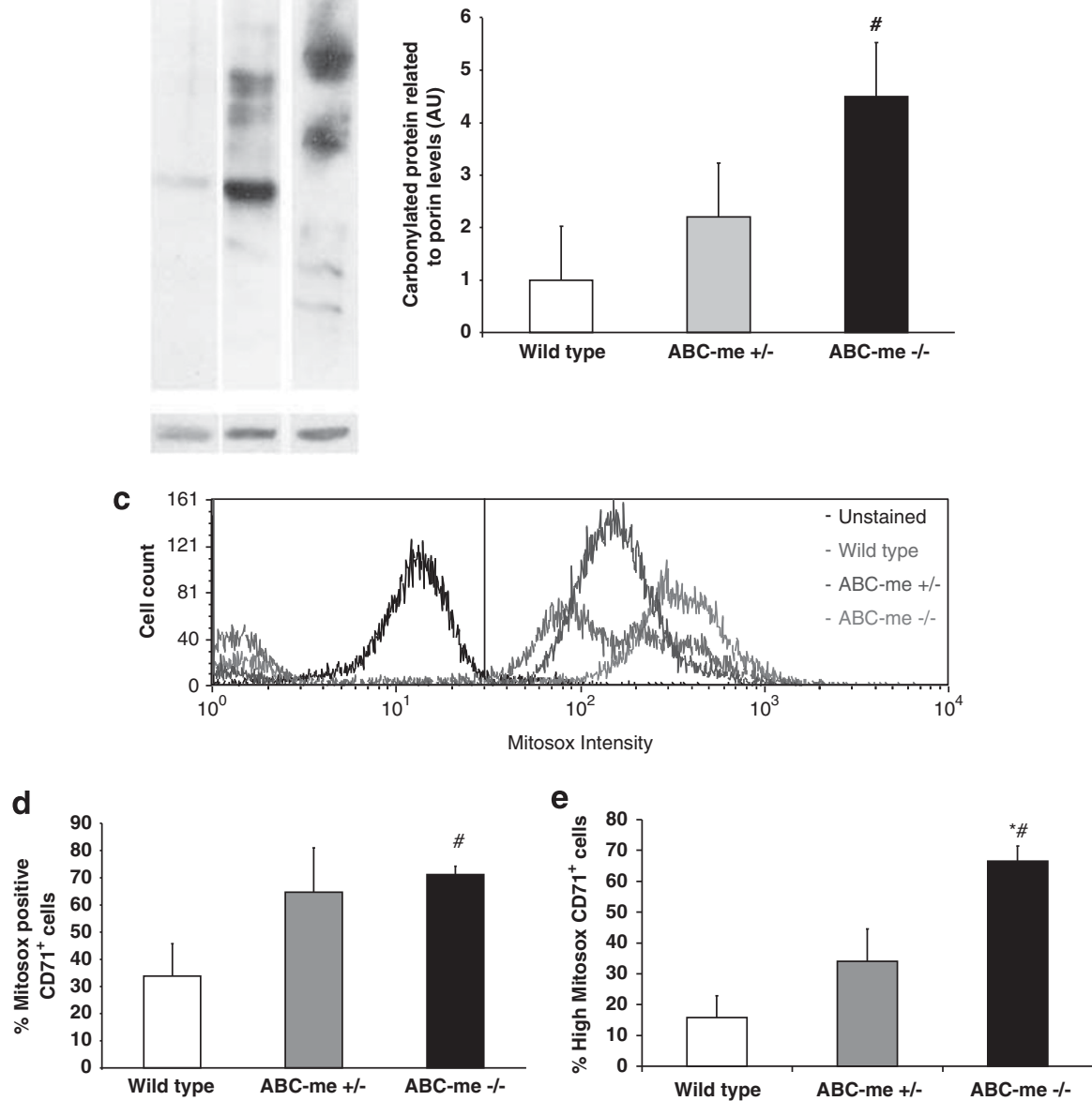

Figure 4 ABC-me-l- blood cells exhibit elevated mitochondrial ROS and oxidative damage. (a) Carbonylated proteins in mitochondrial fractions isolated from WT, ABC$\mathrm{me}+/$ - and $\mathrm{ABC}-\mathrm{me}-/$ - day $10.5 \mathrm{pc}$ embryonic blood. (b) Protein carbonylation was determined by densitometry of 2,4-dinitrophenylhydrazine derivatized carbonyl groups, then detected using anti-2,4-dinitrophenol antibody. Note that ABC-me-l- mitochondria contained significantly more oxidized proteins than WT $(n=3$, \# $P<0.05$ Student's t-test). Error bars representative of standard error. (c) $\mathrm{CD} 71^{+}$cells from day $10.5 \mathrm{pc}$ embryonic blood stained with mitochondrial superoxide probe Mitosox. A representative plot of cell number ( $y$ axis) and Mitosox fluorescence intensity ( $x$ axis) demonstrates an increase in the number of ABC-me- $/-$ cells with high values Mitosox fluorescence intensity. (d) Quantification of percentage of $\mathrm{CD} 71^{+}$cells positive for Mitosox staining (right side of the black bar, gated using unstained cells). ABC-me-l- exhibits significantly elevated percentage of Mitosox-positive cells in comparison with WT (\# $n=3$ experiments; $1 \times 10^{5}$ cells per sample; $P<0.05$ ANOVA.). Error bars representative of standard deviation. (e) Quantification of percentage of $\mathrm{CD} 71^{+}$cells exhibiting high Mitosox fluorescence. These were identified as erythroid precursors showing a value of Mitosox fluorescence intensity that is above the peak of $A B C$-me- $/$ - cells count. ABC-me- $/$ - samples contain increased percentage of cells with high Mitosox fluorescence as compared with WT (\#) and ABC-me $+/-{ }^{*} ; n=3$ experiments; $1 \times 10^{5}$ cells per sample; $P<0.05$ ANOVA). Error bars representative of standard deviation 
colonies formed reflects the initial number of surviving progenitor cells together with their ability to differentiate. After 5 days of differentiation, ABC-me-/- embryonic blood progenitors formed approximately 20 colonies per plate, whereas WT and ABC-me+/- blood generated approximately 60 colonies (Figure 5a). Furthermore, ABC-me-/-
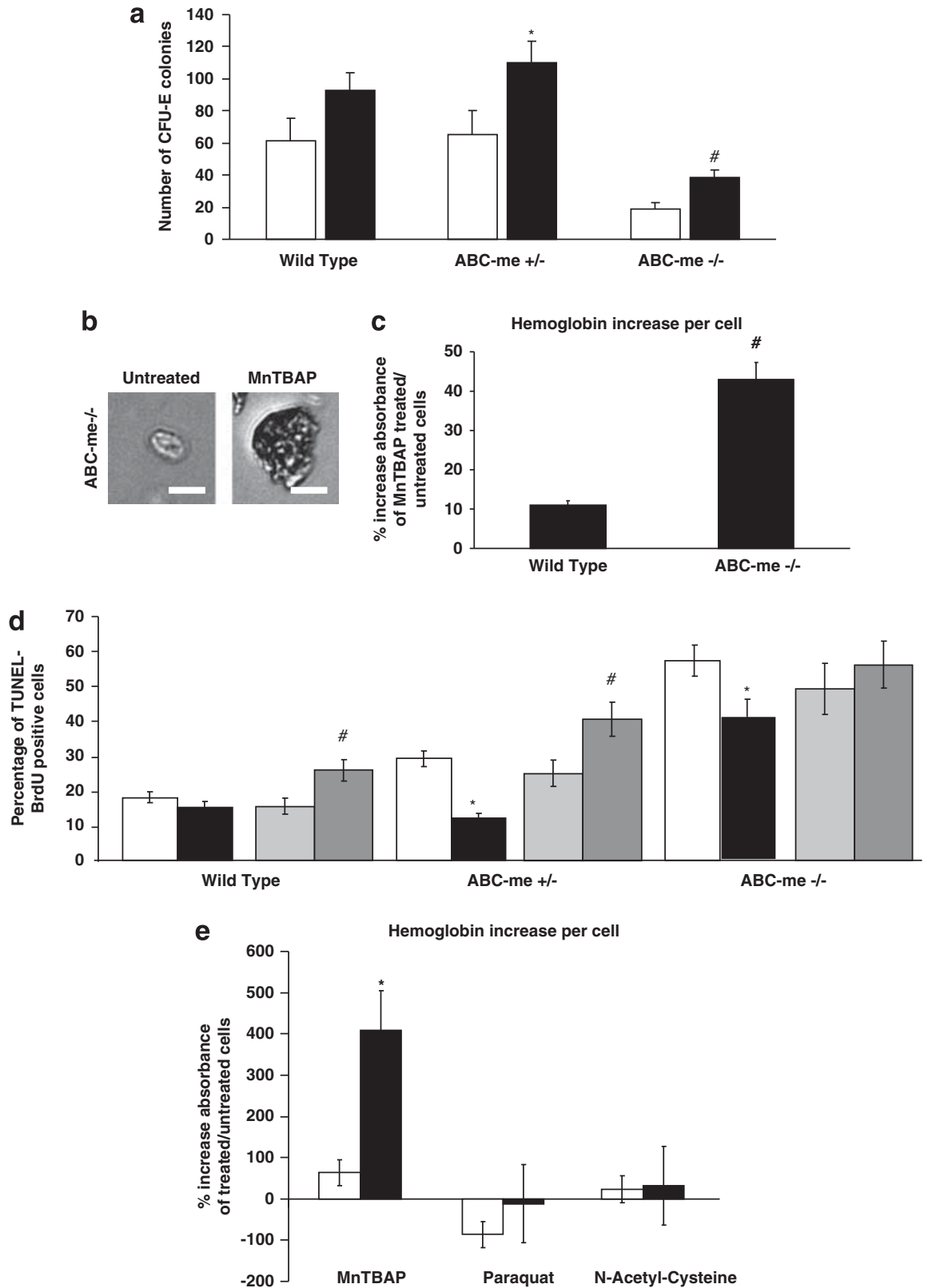

Figure 5 Antioxidant treatment partially rescues ABC-me-/- erythroid development and hemoglobinization. (a) Isolated day $10.5 \mathrm{pc}$ embryonic blood differentiated in vitro in erythroid colony assay media for 5 days was treated with $200 \mu \mathrm{M}$ of the SOD2 mimetic MnTBAP at day 0. MnTBAP significantly increased the number of CFU-E colonies present (black bars) per plate over untreated controls (open bars) in WT, ABC-me $+I-\left(^{*}\right)$ and ABC-me $-I-(\#)(n=5$ experiments; all samples scored in duplicate; multiple examiners used, $P<0.05$ ANOVA). (b) Images of day 5 CFU-E colonies in a methyl-cellulose erythroid colony assay. Note the reduced size, transparency and abnormal cellularity of the ABC-me-/ - colony in comparison with those treated with $200 \mu \mathrm{M} \mathrm{MnTBAP}$ (image representative of multiple CFU-E colonies from five independent experiments). Scale bar, $20 \mu \mathrm{m}$. (c) ABC-me-l-colonies treated with $200 \mu \mathrm{M}$ MnTBAP showed significantly increased levels of hemoglobin per cell as detected by the increase in absorbance (percentage of absorbance obtained from MnTBAP-treated cells over untreated cells) corrected by cell number ( $n=3$ independent experiments; WT versus ABC-me-1-; \# $P \leqslant 0.05$ ANOVA). (d) Effects of anti- and pro-oxidants on apoptosis of ABC-me-1- erythroid progenitors. Isolated day 10.5 embryonic blood cells were differentiated in vitro for 5 days in the presence of $200 \mu \mathrm{M}$ MnTBAP (black bars) or $20 \mu \mathrm{M}$ of the oxidant Paraquat (PQ, gray bars) or the corresponding vehicles (open bars for MnTBAP, light gray for $\mathrm{PQ}$ ). Apoptosis was determined by flow cytometry analysis of TUNEL-BrdU-stained cells detected with an anti-BrdU antibody conjugated to FITC. ABC-me- $/$ - and ABC-me $+/$ - cells treated with MnTBAP exhibited significantly reduced level of apoptosis when compared with untreated $\left({ }^{*}\right)$. Paraquat significantly increased apoptosis only in WT and ABC-me $+1-$ cells (\# $n=4$ experiments performed in duplicate; $P \leqslant 0.05$ ANOVA). (e) The effect of anti- or pro-oxidant treatments on hemoglobinization of $A B C-m e+I-$ (open bars) and ABC-me- $I-$ (black bars) ES cells differentiated in vitro in an erythroid colony assay. The pro-oxidant paraquat and the scanvenger antioxidant NAC did not have significant effects on hemoglobin synthesis. ABC-me-/- cells treated with $200 \mu \mathrm{M}$ MnTBAP demonstrated significantly increased levels of total hemoglobin per cell as detected by absorbance over ABC-me $+I-\left({ }^{*} ; n=3 ; P \leqslant 0.05\right.$ ANOVA). Error bars representative of standard deviation 
colonies were smaller and more transparent (denoting lack of hemoglobinization; Figure $5 b$ ). On the other hand, the mitochondrial antioxidant MnTBAP increased by nearly twofold the formation of colonies in ABC-me-/- embryonic blood (40 colonies MnTBAP versus 20 colonies untreated). Although this data illustrated that MnTBAP fostered the survival of $A B C-m e-/-$ erythroid progenitors (closer to the 60 colonies detected in WT untreated), this fold increase was similar (approximately twofold) in WT and ABC-me-/- blood cells (Figure $5 \mathrm{a}$ ). Therefore, the reduction of mitochondrial ROS levels can improve erythroid progenitor survival in all backgrounds. However, MnTBAP specifically rescued the aberrant phenotype of ABC-me-/- colonies, showing a bigger size and hemoglobinization (Figure $5 b$ ). To further confirm the rescue in hemoglobin synthesis, we measured the increase in hemoglobin levels per cell after MnTBAP treatment both in WT and ABC-me-/- colonies (Figure 5c). Wildtype cells treated with MnTBAP showed a modest increase in hemoglobin levels per cell $(10 \%)$, whereas MnTBAP-treated ABC-me-l- cells showed a $40 \%$ increase in hemoglobin (fourfold over the WT). Altogether, these experiments show that MnTBAP specifically restored the capacity of surviving ABC-me-/- erythroid progenitors to differentiate and synthesize hemoglobin.

To address whether apoptosis was also responsible for these defects in ex-vivo differentiation caused by the deletion of ABC-me, we stained dispersed colonies with TUNEL-BrdU and analyzed them by flow cytometry. ABC-me-l- (55\%) differentiated cells were apoptotic, a higher percentage than detected in ABC-me $+/-$ (around $30 \%$ ) and WT cells (19\%; Figure $5 \mathrm{~d}$ ). Of note, MnTBAP significantly reduced the percentage of apoptotic cells in ABC-me-/- (to 40\%) and in ABC-me $+/-$ (to 12\%), but not in WT cells (Figure $5 \mathrm{~d}$ ). On the other hand, the addition of the pro-oxidant paraquat only increased apoptosis in WT (19\% untreated cells versus $30 \%$ paraquat-treated cells) and ABC-me $+1-$ cells $(30 \%$ untreated versus $48 \%$ paraquat-treated cells), but not in ABC-me-1- cells (Figure $5 \mathrm{~d}$ ). The lack of a mitochondrial pro-oxidant-induced apoptosis in ABC-me-/- cells suggests that the lack of $A B C$-me fully activates the apoptotic response associated with oxidative stress (Figure $5 \mathrm{~d}$ ). Interestingly, a mainly cytosolic ROS scavenger ( $N$-acetyl-cysteine, NAC) did not induce any significant increase in hemoglobin levels per cell of ABC-me-/- erythroid cells differentiated ex vivo (Supplementary Figure S3).

To further confirm these ex-vivo rescues in hemoglobin synthesis by MnTBAP and the erythroid cell autonomous defect of ABC-me loss-of-function, we also used ABC-me $+/$ - and ABC-me-I- ES cells differentiated to the erythroid lineage. To differentiate these cells, dispersed embryonic bodies obtained from $A B C-m e+/-$ and ABC-me-/- ES cells were also subjected to the erythroid colony assay, together with distinct treatments. MnTBAP treatment induced a remarkable $400 \%$ increase in hemoglobin levels in the erythroid cells derived from ABC-me-/-ES cells as compared with only $60 \%$ increase in hemoglobin in cells derived from the ABC-me $+/-$ ES cells (Figure $5 e$ ). Consistent with the ex-vivo data, NAC did not increase the hemoglobin levels in differentiated ABC-me-I- ES cells (Figure $5 e$ ). On the other hand, treatment with the mitochondrial pro-oxidant paraquat caused a tendency to decrease hemoglobin levels in ABC-me + /- differentiated ES cells and no changes in the low hemoglobin levels detected in ABC-me-/--differentiated ES cells (Figure $5 \mathrm{~d}$ ).

\section{Discussion}

We have identified that the mitochondrial transporter ABC-me is required for erythrocyte development. We show that the lack of $A B C$-me function causes a profound decrease in the erythrocyte precursor cell population by triggering apoptosis. Deletion of ABC-me causes severe anemia by day $10.5 \mathrm{pc}$ and mouse embryonic lethality by day $12.5 \mathrm{pc}$. This lethality confirms the physiological relevance of the previous findings showing that $A B C$-me was exclusively detected in the first hematopoietic sites during development (yolk sac blood islands, during embryonic day $10.5 \mathrm{pc}$ ). Of note, ABC-me-/embryo phenotype has important similarities with GATA-1-/mice (ABC-me transcriptional regulator) phenotype. GATA-1 deletion causes severe anemia at day 10.5 pc embryos, such as in ABC-me-/- embryos. Furthermore, GATA-1-/- embryonic blood shows impaired differentiation beyond the proerythroblast stage (such as in ABC-me-/- embryos) and erythroid colony assays of GATA-1-/- ES cells show colonies lacking hemoglobin (such as we report here in isolated embryonic blood cells from ABC-me-/- mice).${ }^{18,19}$ ABC-me joins the list of GATA-1 downstream factors that are required for erythroid differentiation. Interestingly, the GATA-1 downstream factor B-cell lymphoma extra large (BCL-xL), a mitochondrial antiapoptotic protein, was shown to be dispensable for primitive erythropoiesis. $^{20,21}$ In this regard, deletion of BCL-x did not increase apoptosis during primitive erythropoiesis, ${ }^{21}$ in marked contrast to ABC-me deletion. Thus, our study identifies, for the first time, that $A B C$-me is a previously unknown mitochondrial factor downstream of GATA-1 required to prevent apoptosis during primitive erythropoiesis.

The next set of experiments addressed the mechanism by which the lack of ABC-me causes apoptosis in erythroid cells. In this regard, we find that $A B C$-me deletion caused oxidative stress, by increasing mitochondrial ROS (specifically, mitochondrial superoxide levels) and mitochondrial protein oxidation in erythroid cells. Oxidative stress was sufficient to trigger apoptosis, as MnTBAP (a catalytic antioxidant of the mitochondrial matrix, SOD2 mimetic), rescued to some extent the apoptotic phenotype in erythroid cells. Given the previous data demonstrating the role of ABC-me in hemoglobin synthesis and protection from oxidative stress, ${ }^{12,13}$ we propose that defects caused by $\mathrm{ABC}-\mathrm{me}$ deletion are a consequence of the roles played by $A B C-m e$, both in protecting from mitochondrial oxidative stress and regulating heme synthesis. In addition, this protective role of ABC-me cannot be entirely explained by its capacity to stabilize the mitochondrial iron importer Mfrn1. This hypothesis is supported by the following evidence: the lack of ABC-me would reduce Mfrn1 protein levels, which in turn would reduce mitochondrial iron uptake. Therefore, it is unlikely that increased mitochondrial oxidative stress would be caused by mitochondrial iron overload, as the iron import should be decreased in ABC-me-/- erythroid cells. Indeed, flow cytometry data of ABC-me-/- surviving cells show lower 
levels of CD71 expression, suggesting less import of iron to the cell and to the mitochondria to synthesize hemoglobin (Supplementary Figure S4). Furthermore, the lack of rescue by NAC, (an antioxidant with lower efficiency in the mitochondria), together with the absence of increased protein carbonylation in total lysates, suggests that other subcellular compartments do not show a marked increase in oxidative stress. These data are complementary to the treatment with a mitochondrial pro-oxidant (paraquat), which is unable to further stimulate apoptosis in ABC-me-/- cells.

The rescue of apoptosis by MnTBAP is not complete in ABC-me-1- cells. This result suggests that increased superoxide production in the mitochondria might not be the unique contributor to apoptosis. Therefore, a reduction in an additional unknown function of $\mathrm{ABC}$-me beyond oxidative stress handling and related to hemoglobin synthesis could also be contributing to apoptosis. It is likely that this function related to hemoglobin synthesis would be partly through Mfrn1 stabilization. Indeed, we detect a specific reduction in Mfrn1 protein in ABC-me-/- embryos (see Supplementary Figure S1).

We have recently reported that $A B C$-me protects from oxidative stress induced by ischemia-reperfusion in cardiac cells. ABC-me $+/-$ hearts showed decreased protection from oxidative stress in the absence of changes in Mfrn1 protein levels. ${ }^{13}$ This further demonstrates the existence of a conserved function of $\mathrm{ABC}$-me preventing oxidative damage independent of its role stabilizing Mfrn1.

Altogether, the demonstration that $A B C$-me is essential for erythroid differentiation in mice identifies a new possible target of orphan blood diseases showing defective erythrocyte differentiation (either genetic or drug-induced). In addition, our work also shows that antioxidant treatment could help to treat blood disorders potentially related to $A B C-m e$ inactivation.

\section{Materials and Methods}

Animals. $A B C-m e+1-$ mice were generated by a gene-targeted $\mathrm{KO}$ strategy by Lexicon Genetics on a C57BL6/129SvEvBrd mixed background and backcrossed up to four generations onto C57BL6 background. All procedures and experiments were carried out according to the institutional guidelines for animal care at the Tufts University and Boston University in compliance with the United States Public Health Service regulations.

Embryonic blood isolation and May-Grunwald-Giemsa staining. Embryonic blood was isolated from day 10.5 pc embryos, by surgical separation of embryo and yolk sac from maternal tissue, and by their subsequent bleeding and flushing in chilled PBS with heparin. Isolated embryonic blood was fixed in $6 \%$ paraformaldehyde (Sigma, St. Louis, MO, USA) for $1 \mathrm{~h}$ washed in $70 \%$ ethanol, mounted on slides following cytospine and then stained with May-Grunwald-Giemsa for morphological analysis of hematopoietic lineages.

Histology and Immunofluoresence. Surgically isolated embryos were fixed in Botulinum Toxin (Sigma) for $24 \mathrm{~h}$ and kept in $70 \%$ ethanol. Intact embryos were paraffin-embedded, sectioned and stained with $\mathrm{H} \& \mathrm{E}$, benzidine or Okajima staining for analysis of cell number, morphology and hemoglobin content according to standard protocols. For immunofluorescence, intact isolated embryos were flashfrozen in mounting media. Mounted sections of frozen tissue were first dehydrated in acetone, then processed and stained with a FITC-conjugated antibody to detect TUNEL-BrdU and DAPI nuclear stain. TUNEL-BrdU labels damaged DNA and can be detected using an anti-BrdU antibody conjugated to FITC. DAPI stains all types of DNA. Images were analyzed using Image J software (NIH, Bethesda, MD, USA) on a Zeiss (Thornwood, NY, USA) inverted fluorescence microscope.
Cell lines. $A B C-m e+1-$ ES cells were generated by targeted KO strategy by Lexicon Genetics. ES Cells were maintained and expanded in ES cell media (Stem Cell Technologies, Seattle, WA, USA), in the presence of mLIF (Stem Cell Technologies), on a monolayer of growth inactivated murine embryonic fibroblasts (Chemicon Intl Millipore, Temecula, CA, USA). ABC-me-/- ES cells were selected after treatment with geneticin (Sigma) for 2 weeks, then expanded and genotyped. In vitro hematopoietic differentiation of ES cells was carried out as previously described and detailed in the Stem Cell Technologies Technical Manual (and briefly discussed in the colony assay chapter).

Colony assay. Isolated embryonic blood or dispersed embryonic bodies generated from ES cells $\left(1-5 \times 10^{5}\right)$ were added to MethoCult complete hematopoietic media with cytokines (EPO, IL-6, c-KIT; Stem Cell Technologies). All samples were analyzed in duplicate in each experiment. Formation of CFU-E colonies was scored under multiple conditions and was analyzed both in terms of number, size and morphology of the colonies. Samples were obtained after differentiation of these colonies and apoptosis was analyzed using flow cytometry (TUNEL-BrdU, as described below for embryonic blood) and also for hemoglobin content by measuring absorbance (after lysis of colonies) as previously described. ${ }^{6,17}$

Fluorescence-activated cell sorting (FACS) analysis. Isolated embryonic blood from day $10.5 \mathrm{pc}\left(\sim 1 \times 10^{5}\right)$ was immunostained simultaneously with PE-conjugated anti-TER119 (1:100) (BD Pharmingen, San Diego, CA, USA) and FITC-conjugated anti-CD71 (Transferrin receptor) (1:100; BD Pharmingen). For apoptosis analysis, PE-conjugated anti-CD71 $(1: 100)$ either with FITC-conjugated anti-TUNEL-BrdU $(1: 100)$, anti-Annexin V ( $3: 100)$ (APC-conjugated, BD Pharmingen) or the caspase 3 FLICA (FAM-VAD-FMK) substrate (Immunochemistry Technologies, LLC, Bloomington, MN, USA) was used according to the manufacturer's instructions. Flow cytometry was performed using a FACS Calibur and LSRII (Becton Dickinson, San Jose, CA, USA) and R1-R5 gating was selected as previously described. ${ }^{15,22}$ FACS data analysis was performed with the FCS Express V3 software, Los Angeles, CA, USA. Cell debris was excluded by gating on the forward and side scatter plot.

Mitochondrial isolation. Mitochondrial isolation from embryonic blood was achieved using a mitochondrial isolation kit for tissue (Pierce, Rockford, IL, USA) following the manufacturer's instructions.

Protein oxidation detection. To detect oxidized proteins, we used the Oxyblot protein oxidation detection kit (Chemicon Intl) according to the manufacturer's manual. It is based on detection of carbonyl groups that are introduced into protein side chains when proteins are exposed to oxidative stress. These carbonyl groups are derivatized with dinitrophenylhydrazine, which then can be detected using specific antibodies by western blot.

ROS detection. Isolated embryonic blood was collected as described and $1 \times 10^{6}$ cells were stained with $5 \mu \mathrm{M}$ Mitosox (Invitrogen, Eugene, OR, USA) for $30 \mathrm{~min}$ at $37^{\circ} \mathrm{C}$. Mitosox-stained samples were also treated simultaneously with $10 \mu \mathrm{M}$ Verapamil (Sigma-Aldrich, St. Louis, MO, USA), as a control to avoid differences in staining due to hypothetical different multi-drug resistance activity of the cells. In addition, samples (duplicates) were also stained with $10 \mu \mathrm{M}$ TMRE (Invitrogen), as Mitosox loading is dependent on mitochondrial membrane potential (no differences in TMRE staining were detected in all measurements performed). After the incubation time, cells were washed twice in warm PBS-1\% BSA and immunostained with FITC-conjugated anti-CD71 antibody, as described above, and analyzed by FACS. Unstained cells (no Mitosox and no FITC anti-CD71) were used as a control to detect the specific Mitosox signal.

Western blot. Samples were prepared from cell lysates or mitochondrial fractions, loaded in $12 \%$ polyacrylamide gel and transferred onto PVDF membrane using a semi-dry transfer machine (Bio-Rad, Hercules, CA, USA). ABC-me antibody was diluted 1/2000 and used as described. ${ }^{6}$ Porin (Calbiochem, San Diego, CA, USA) and $\alpha$-actin (Novus Biologicals, Littleton, CO, USA) antibodies were used according to manufacturer's instruction. Mfrn1 and BCL-xL (Cell Signalling, Danvers, MA, USA) antibodies were used as previously described ${ }^{10,23,24}$ (Supplementary Figure S1).

Antioxidants and chemicals. Agents used as supplements in in vitro differentiation assays include; Mn(III)TBAP (Cayman Chemical, Ann Harbor, MI, USA), $\mathrm{N}$-acetyl-Cysteine (Sigma) and Paraquat (Sigma). These chemicals (and their vehicles 
in duplicate wells, as control for the treatments) were added to cells in MethoCult complete hematopoietic media when plated (day 0 of differentiation). The final concentrations in the MethoCult media were as follows: $200 \mu \mathrm{M}$ for both NAC and MnTBAP, $20 \mu \mathrm{M}$ for Paraquat (PQ).

Statistical analysis. Data were analyzed by Student's $t$-test or ANOVA $(\alpha=0.05)$ with the GraphPad Prism software (La Jolla, CA, USA).

\section{Conflict of Interest}

The authors declare no conflict of interest.

Acknowledgements. We are grateful to Drs. Alex Sheftel, Prem Ponka, Solomon Graf, Carlo Brugnara, Thomas Langer and Roland Lill for fruitful discussions and advice. We thank Dr. Yuko Fujiwara for her advice on mouse pathology. We are grateful to Barry Paw for providing the Mfrn1 antibody. This work was supported by the National Institutes of Health Grants R01HL071629-03 and R01DK074778 (to OSS). ML is a recipient of a post-doctoral fellowship from Fundación Ramón Areces. We are thankful to Professor Daniel Dagan for comments on the manuscript.

\section{Author Contributions}

$\mathrm{BBH}, \mathrm{ML}, \mathrm{AAE}$ and $\mathrm{LR}$ performed research and analyzed the data; WQ and SEH performed research; ML and BBH wrote the paper; $B B H, A E E, M L$ and OSS designed experiments and analyzed the data. TMS and HKM contributed with vital new reagents and analytical tools.

1. Krishnamurthy $P$, Xie $T$, Schuetz JD. The role of transporters in cellular heme and porphyrin homeostasis. Pharmacol Ther 2007; 114: 345-358.

2. May BK, Dogra SC, Sadlon TJ, Bhasker CR, Cox TC, Bottomley SS. Molecular regulation of heme biosynthesis in higher vertebrates. Prog Nucleic Acid Res Mol Biol 1995; 51: 1-51.

3. Ponka P. Tissue-specific regulation of iron metabolism and heme synthesis: distinct control mechanisms in erythroid cells. Blood 1997; 89: 1-25.

4. Bottomley SS. Congenital sideroblastic anemias. Curr Hematol Rep 2006; 5: 41-49.

5. Nilsson R, Schultz IJ, Pierce EL, Soltis KA, Naranuntarat A, Ward DM et al. Discovery of genes essential for heme biosynthesis through large-scale gene expression analysis. Cell Metab 2009; 10: 119-130.

6. Shirihai OS, Gregory T, Yu C, Orkin SH, Weiss MJ. ABC-me: a novel mitochondrial transporter induced by GATA-1 during erythroid differentiation. EMBO J 2000; 19 : 2492-2502.
7. Graf SA, Haigh SE, Corson ED, Shirihai OS. Targeting, import, and dimerization of a mammalian mitochondrial ATP binding cassette (ABC) transporter, ABCB10 (ABC-me) J Biol Chem 2004; 279: 42954-42963.

8. Fraser ST, Isern J, Baron MH. Maturation and enucleation of primitive erythroblasts during mouse embryogenesis is accompanied by changes in cell-surface antigen expression. Blood 2007; 109: 343-352.

9. Kina T, Ikuta K, Takayama E, Wada K, Majumdar AS, Weissman IL et al. The monoclonal antibody TER-119 recognizes a molecule associated with glycophorin A and specifically marks the late stages of murine erythroid lineage. Br J Haematol 2000; 109 280-287.

10. Chen W, Paradkar PN, Li L, Pierce EL, Langer NB, Takahashi-Makise N et al. Abcb10 physically interacts with mitoferrin-1 (SIc25a37) to enhance its stability and function in the erythroid mitochondria. Proc Natl Acad Sci USA 2009; 106: 16263-16268.

11. Lill R, Kispal G. Mitochondrial ABC transporters. Res Microbiol 2001; 152: 331-340.

12. Chloupkova M, LeBard LS, Koeller DM. MDL1 is a high copy suppressor of ATM1: evidence for a role in resistance to oxidative stress. J Mol Biol 2003; 331: 155-165.

13. Liesa M, Luptak I, Qin F, Hyde BB, Sahin E, Siwik DA et al. Mitochondrial transporter ATP binding cassette mitochondrial erythroid is a novel gene required for cardiac recovery after ischemia/reperfusion. Circulation 2011; 124: 806-813.

14. Young $L$, Leonhard K, Tatsuta T, Trowsdale J, Langer T. Role of the $A B C$ transporter Mdl 1 in peptide export from mitochondria. Science 2001; 291: 2135-2138.

15. Zhang J, Socolovsky M, Gross AW, Lodish HF. Role of Ras signaling in erythroid differentiation of mouse fetal liver cells: functional analysis by a flow cytometry-based novel culture system. Blood 2003; 102: 3938-3946.

16. Martin FM, Bydlon G, Friedman JS. SOD2-deficiency sideroblastic anemia and red blood cell oxidative stress. Antioxid Redox Signal 2006; 8: 1217-1225.

17. Elorza A, Hyde B, Mikkola HK, Collins S, Shirihai OS. UCP2 modulates cell proliferation through the MAPK/ERK pathway during erythropoiesis and has no effect on heme biosynthesis. J Biol Chem 2008; 283: 30461-30470.

18. Pevny L, Lin CS, D'Agati V, Simon MC, Orkin SH, Costantini F. Development of hematopoietic cells lacking transcription factor GATA-1. Development 1995; 121 163-172.

19. Weiss MJ, Orkin SH. Transcription factor GATA-1 permits survival and maturation of erythroid precursors by preventing apoptosis. Proc Natl Acad Sci USA 1995; 92: 9623-9627.

20. Gregory T, Yu C, Ma A, Orkin SH, Blobel GA, Weiss MJ. GATA-1 and erythropoietin cooperate to promote erythroid cell survival by regulating bcl-xL expression. Blood 1999 94: 87-96

21. Motoyama N, Wang F, Roth KA, Sawa H, Nakayama K, Nakayama K et al. Massive cell death of immature hematopoietic cells and neurons in Bcl-x-deficient mice. Science 1995 267: $1506-1510$.

22. Rooke HM, Orkin SH. Phosphorylation of Gata1 at serine residues 72,142 , and 310 is not essential for hematopoiesis in vivo. Blood 2006; 107: 3527-3530.

23. Chen W, Dailey HA, Paw BH. Ferrochelatase forms an oligomeric complex with mitoferrinand Abcb10 for erythroid heme biosynthesis. Blood 2010; 116: 628-630.

24. Liesa M, Borda-d'Agua B, Medina-Gomez G, Lelliott CJ, Paz JC, Rojo M et al Mitochondrial fusion is increased by the nuclear coactivator PGC-1beta. PLoS One 2008 3. $\mathrm{e} 3613$.

Supplementary Information accompanies the paper on Cell Death and Differentiation website (http://www.nature.com/cdd) 